\title{
Effect of composition and processing conditions on selected characteristics of extruded corn instant gruels enriched with fruits addition
}

\author{
Magdalena Kręcisz ${ }^{1}$, Agnieszka Wójtowicz ${ }^{1, *}$, and Anna Oniszczuk $^{2}$ \\ ${ }^{1}$ University of Life Sciences, Doświadczalna 44,20-280 Lublin, Poland \\ ${ }^{2}$ Medical University of Lublin, Chodźki 4a, 20-093 Lublin, Poland
}

\begin{abstract}
Cranberries and goji berries were used as natural supplements in extruded instant corn gruels. The effects of additive type and level, as well as extrusion-cooking screw speed on selected properties of extrudates were tested. Corn grit was used as the base raw materials and dry and ground goji berries and cranberries were added at 1,2,3,4 and 5\%. Extrusion-cooking of blends was performed with a single screw extruder at temperature ranged $125-135^{\circ} \mathrm{C}$ using various screw speed during processing. Extrudates were ground below $1 \mathrm{~mm}$ for instant gruels. Water absorption, water solubility, as well as colour profile were tested. The results showed that the highest water absorption was evaluated for instant gruels consist the highest amount of goji berries extruded at the highest screw speed during processing, while cranberries addition have no significant effect on water absorption. Increasing amount of dry cranberries and goji berries in the recipe affected on lowering the water solubility of the extrudates. Significant effect of fruits on color coordinates was observed. Increasing amount of dry berries lowered lightness $\mathrm{L}^{*}$ and yellowness of instant gruels, especially when goji berries were used in the recipe. Increasing the screw speed during processing decreased intensity $b^{*}$ values.
\end{abstract}

\section{Introduction}

The extrusion-cooking technique seems to be a milestone in the production of instant products according to processing simplicity [1]. Full automatization of processing compacted lines and limitation of stuff contact with foods, offering the high hygienic and sanitary requirements extremely important in baby and infant instant food production. The main equipment in processing line is single or twin-screw extruder useful for thermomechanical treatment of cereal raw materials as corn, rice, buckwheat or wheat flour with various additives into extrudates with unique characteristics [26]. Equipment needed for processing of instant gruels and baby food is easy to operate, energy saving and more efficient comparing to conventional process with drum drying of cooked cereal slurries. Configuration and operational parameters of extruder make it possible to create products' specific characteristics as viscosity, density, water absorption or instant gruels consistency with water or milk [7]. These features are very important for ready-to-eat (RTE) extrudates as well as for extruded components used in bakery, beverages, chemicals or pharmaceutical products or biodegradable thermoplastic starch $[8,9]$.

The functional properties of extruded foods plays an important role for their acceptability and properties as water absorption, water solubility, oil absorption indexes, expansion index, bulk density and viscosity of the dough $[10,11]$. Raw materials composition, especially carbohydrates and protein content, as well as fibrous fractions of food components, can affect on several physiochemical characteristics of extruded foodstuffs $[1-3,5,7,10,11]$. There is a possibility to improve both nutritional and quality characteristics of food by supplementation with some fruits addition [12].

Cranberries and goji berries are nowadays popular additives for food supplementation according to unique composition of these berries.

Cranberry according to its excellent nutritional characteristic is qualified as one of the "superfruits". The chemical composition of cranberry depends on the variety, crop region and storage conditions. Ripped cranberry consists $88 \%$ of water, $4.2 \%$ of sugar and organic acids, $1.2 \%$ of pectin, minerals (potassium, calcium, sodium, selenium), vitamins of A, C, E and B group, flavonoids and anthocyanins, catechins and proanthocyanins [13-15]. Cranberries are valuable diet component known from ancients and used, for example by Indians, in natural medicine for cold, whoopingcough, scurvy, rheumatism, gastrointestinal problems, oral mucositis, angina, urinary tract infection and many others $[13,16]$. Cranberries have the potential ability to limit the development and severity of certain cancers and vascular diseases including atherosclerosis, ischemic

\footnotetext{
Corresponding author: agnieszka.wojtowicz@up.lublin.pl
} 
stroke, and neurodegenerative diseases of aging $[17,18]$. Research results confirm positive effects of cranberries for prevent the bacteria expansion in urinal tract, antibacterial and antimicrobial effect, anti-cancer, antiinflammatory, protect against food poisoning e.g. due to E. coli, inhibit adhesion of Helicobacter pylori, a major cause of gastric cancer, to human gastric mucus, thereby prevent ulcerative disease. NDM (the nondialyzable material) in cranberries, contains about $65 \%$ proanthocyanidins, along with a much smaller quantity $(0.35 \%)$ of anthocyanins, is helpful in treatment of mouth diseases and cold, NDM is limiting growth of bacteria causing gum disease and tooth decay, and also protecting from problems with fungi disorders [15, 19, $20]$.

More and more popular component in functional and pro-healthy foods are goji berries, growing as fruits of Lycium barbarum. According to the high amount of biologically active components also the goji berry is called as "superfruit". Its caloric value is $348 \mathrm{kcal}$ in 100 $\mathrm{g}$ of dry berries [21]. Goji berries are used as medicinal in various diseases. The medical power of these fruits was proved by scientists from China since over 2000 years.. The most of the research was based on its antioxidant characteristics because of 15-times higher antioxidant potential than of green tea [22]. Goji berries are characterized red-orange colour formed by carotenoids [23]. One of the most important components of goji berries are polyphenols with high antioxidant activity [24]. The biggest group of components are compexed polysaccharides, and moreover vitamins as ascorbic acid, tiamin, ryboflavin or glucopyranosids. It can be eaten as dry or cooked fruits. In these forms, it could be used for tea, soups, desserts, or other meals [25]. A unique characteristic of goji berry is the effect of the presence of 20 types of amino acids, including 8 of exogenous. These essential amino acids play the important role in human body, i.e. lysine is playing the role in antibodies production, is the component of collagen and supports $\mathrm{B}_{3}$ vitamin; isoleucine is supporting in haemoglobins production, and methionine causes acidity of urine and destroy bacteria. Goji berries are good source of mineral components, one of the biggest natural source of iron, consist also calcium, chromium, germanium, magnesium, potassium, zinc or selenium [26].

Some results showed the reduction of fibroids (myoma) growing and production of antibodies by spleen in the experiments on mice feeding with polysaccharide complex from goji berries. Additionally, the increase of lymphocytes production and decrease of fat oxidation was observed on mice infected with fibroids [19]. It was proven that components of goji berries inhibit growth or even kill cancer cells. Tang et al. [27] confirmed the results of previous research showed that the consumption of juice from goji berries increased lymphocytes, interleukin-2 and immunoglobulin $G$ levels. Some research confirmed the anti-cancer effect of scopoletin and 2-O- $\beta-D-$ glucopyranosyl-L-ascorbic acid present in goji berries [28]. The extract from goji berries consist taurine may inhibit progression of eye diabetic retinopathy. High content of bioactive components in goji berries can protect neurons in eye retina in diabetic animals from destroying by oxygen free radicals, excessive amount of glucose and kainic acid [29]. In 2012 it was proved protective effect of goji extracts on human retina nerve cells [30]. Goji berries characterise antioxidant activity thanks to the content of antioxidants, carotenoids, flavonoids and polysaccharide complexes and analogues of vitamin C. Antioxidant capacity of fresh goji berries is comparable with cherries and redcurrant. Goji extract is also affecting on lowering the activity of liver enzymes causing hepatic necrosis when consumed with alcoholic beverages [31].

Presented advantages of cranberry and goji berry suggest that they may be utilized for supplementation of food products enhancing the nutritional characteristics and creating new type of functional foods. So, the aim of this study was the evaluation of the effect of goji berries and cranberries supplementation of selected characteristics of corn-based instant gruels processed with the extrusion-cooking.

\section{Materials and Methods}

\subsection{Sample preparation}

Corn grit (purchased from PZZ Lubella Sp. z o. o. Sp. K., Lublin, Poland) was used as the base raw material and dry and ground goji berries and cranberries were added in amount of 1, 2, 3, 4 and 5\% of weight. Raw materials were mixed using a ribbon mixer and moistened with a specific volume of tap water to obtain the moisture content before the extrusion-cooking at the level of $14 \%$ [32]. The mixing time was set for 15 minutes to obtain a loose structure. The mixtures of raw materials were rested for 1 hour to unify the moisture. The moisture content of raw materials, moistened mixtures and extrudates was checked by the drying method [33] by drying at $130^{\circ} \mathrm{C}$ for 1 hour using air dryer SWL-53 SDT (Pol-Eko-Aparatura, Wodzisław Śląski, Poland).

\subsection{Extrusion-cooking of instant gruels}

The prepared mixtures were fed into the extruder's hopper. The extrudates were processed using a singlescrew extruder TS-45 (L/D=12:1). The range of the temperatures of the extrusion-cooking process was as follows: $125 / 130 / 135^{\circ} \mathrm{C}$, respectively in three extruder's sections. The process was carried out by modifying the rotational screw speed between 80,100 and $120 \mathrm{rpm}$. A forming die with a single open of $3 \mathrm{~mm}$ in diameter was used. After extrusion extrudates were cooled down and ground with a laboratory grinder LMN10 (TestChem, Radlin, Poland) for a granulation below $1 \mathrm{~mm}$.

\subsection{Physical properties}

\subsubsection{Water absorption index (WAI)}


Water absorption index (WAI) was determined with centrifugation method [34] for each sample in three replications. In brief, $0.7 \mathrm{~g}$ of extruded gruels was mixed with $7 \mathrm{~mL}$ of water for $10 \mathrm{~min}$ and then centrifuged at rotational speed $15000 \mathrm{rpm}$ for $10 \mathrm{~min}$ in T24D type centrifuge (Leipzig, Germany). The supernatant was removed immediately, the remaining gel was weighted and the WAI was calculated as:

$$
\mathrm{WAI}=\mathrm{W}_{g} / \mathrm{W}_{s} \quad[-]
$$

where: WAI - water absorption index, $\mathrm{w}_{g}$ - weight of gel, $[\mathrm{kg}], \mathrm{w}_{s}$ - weight of dry sample, [kg].

\subsubsection{Water solubility index (WSI)}

Water solubility index (WSI) was determined in triple as solids recovered after total water evaporation at $110^{\circ} \mathrm{C}$ of supernatant obtained from the WAI analysis [35]. Results were calculated from formula:

$$
\mathrm{WSI}=\mathrm{w}_{d s} / \mathrm{w}_{s} \cdot 100[\%]
$$

where: WSI - water solubility index, $\mathrm{w}_{d s}$ - weight of dry solids in supernatant, $[\mathrm{kg}], \mathrm{w}_{s}$ - weight of dry sample, [kg].

\subsubsection{Colour parameters}

Colour measurements were done with the Lovibond CAM-System 500 colorimeter (the Tintometer, UK). CIE-Lab scale was used for evaluation of $L^{*}$ for lightness, $a^{*}$ for $(+)$ redness and (-) greenness, and $b *$ for $(+)$ yellowness and (-) blueness, accordingly. Measurements were performed in 20 replications [9].

\subsubsection{Statistical analysis}

The obtained results were tested with bidirectional ANOVA analysis of variance with interactions and Tukey's test for comparison of means $(p<0.05)$ with the Statistica software (Statistica version 10.0, USA). The first factor was the dry fruits amount (goji berry - G and cranberry - C) and the second was the screw speed (S). RSM (response surface methodology) was used for fitting polynomial models $\left(\mathrm{Y}=\mathrm{b}_{0}+\mathrm{b}_{1} \mathrm{X}_{1}+\mathrm{b}_{2} \mathrm{X}_{2}+\mathrm{b}_{11} \mathrm{X}_{12}\right.$ $+b_{12} X_{1} X_{2}+b_{22} X_{22}$, where $X_{1}$ was the dry fruits amount and $\mathrm{X}_{2}$ was the screw speed applied) and quadratic equations of tested characteristics depend on variables used in the experiment (Table 1).

\section{Results and discussion}

The moisture content of instant gruels ranged from 7.10$7.89 \%$ for corn-goji berry and $6.85-7.83 \%$ for corncranberry gruels (detailed data not shown). These levels of moisture content in extruded corn-fruits instant gruels allow to long term storage, stabile shelf life and microbiological safety of products $[11,36,37]$.

\subsection{Physical properties of corn-goji berry and corn-cranberry instant gruels}

\subsubsection{Water absorption index (WAI)}

The amount of water absorbed by the starch and protein components from corn grits as well as pectin's from goji berries as a gel formed after centrifugation ranged from 5.85 to 6.29 . The results showed that the highest WAI was evaluated for instant gruels with the highest amount of goji berries addition extruded at the highest screw speed during processing (Fig. 1).

Table 1. Adequacy of two variables model fitted for tested

\begin{tabular}{|c|c|c|}
\hline Parameter & Fitted model & $\mathbf{R}^{2}$ \\
\hline \multicolumn{3}{|c|}{ Goji berry } \\
\hline $\begin{array}{c}\text { WAI } \\
{[-]} \\
\end{array}$ & $\begin{aligned} \mathrm{WAI}= & 4.38-0.05 \mathrm{G}+0.04 \mathrm{~S}-0.01 \mathrm{G}^{2} \\
& +0.001 \mathrm{GS}-0.0002 \mathrm{~S}^{2}\end{aligned}$ & 0.21 \\
\hline $\begin{array}{l}\text { WSI } \\
{[\%]}\end{array}$ & $\begin{aligned} \mathrm{WSI}=10.21+1.65 \mathrm{G}+0.07 \mathrm{~S}-0.13 \mathrm{G}^{2} \\
\\
-0.01 \mathrm{GS}+0.0001 \mathrm{~S}^{2}\end{aligned}$ & 0.50 \\
\hline$L^{*}$ & $\begin{array}{c}L^{*}=73.55-6.13 \mathrm{G}+0.10 \mathrm{~S}+0.60 \mathrm{G}^{2} \\
+0.003 \mathrm{GS}-0.0005 \mathrm{~S}^{2}\end{array}$ & 0.95 \\
\hline$a^{*}$ & $\begin{aligned} a^{*}=-9.25+7.56 \mathrm{G}-0.08 \mathrm{~S}-0.84 \mathrm{G}^{2} & \\
& +0.003 \mathrm{GS}+0.0003 \mathrm{~S}^{2}\end{aligned}$ & 0.96 \\
\hline$b^{*}$ & $\begin{array}{c}b^{*}=63.49-2.76 \mathrm{G}+0.17 \mathrm{~S}+0.16 \mathrm{G}^{2} \\
+0.008 \mathrm{GS}-0.001 \mathrm{~S}^{2}\end{array}$ & 0.77 \\
\hline \multicolumn{3}{|c|}{ Cranberry } \\
\hline $\begin{array}{c}\text { WAI } \\
{[-]}\end{array}$ & $\begin{aligned} \mathrm{WAI}= & 7.51-0.07 \mathrm{C}-0.26 \mathrm{~S}-0.03 \mathrm{C}^{2} \\
& +0.002 \mathrm{CS}+0.0001 \mathrm{~S}^{2}\end{aligned}$ & 0.35 \\
\hline $\begin{array}{l}\text { WSI } \\
{[\%]}\end{array}$ & $\begin{aligned} \mathrm{WSI}= & 8.55-1.06 \mathrm{C}+0.18 \mathrm{~S}-0.06 \mathrm{C}^{2} \\
& +0.01 \mathrm{CS}-0.0009 \mathrm{~S}^{2}\end{aligned}$ & 0.29 \\
\hline$L^{*}$ & $\begin{aligned} L^{*}= & 80.43-1.89 \mathrm{C}-0.14 \mathrm{~S}+0.16 \mathrm{C}^{2} \\
& +0.0004 \mathrm{CS}+0.0006 \mathrm{~S}^{2}\end{aligned}$ & 0.49 \\
\hline$a^{*}$ & $\begin{array}{c}a^{*}=-11.17+1.77 \mathrm{C}+0.07 \mathrm{~S}-0.24 \mathrm{C}^{2} \\
+0.0002 \mathrm{CS}-0.0003 \mathrm{~S}^{2}\end{array}$ & 0.32 \\
\hline$b^{*}$ & $\begin{array}{c}\mathrm{b}^{*}=87.05-4.40 \mathrm{C}-0.34 \mathrm{~S}+0.03 \mathrm{C}^{2} \\
+0.02 \mathrm{CS}+0.001 \mathrm{~S}^{2}\end{array}$ & 0.79 \\
\hline
\end{tabular}
characteristics of instant corn gruels supplemented with fruits.

G - Goji; C - Cranberry; S - screw speed; WAI - water absorption index; WSI water solubility index; $\mathrm{L}^{*}$ - lightness, $\mathrm{a}^{*}$ - redness/greenness balance, $b^{*}$ - yellowness/blueness balance,

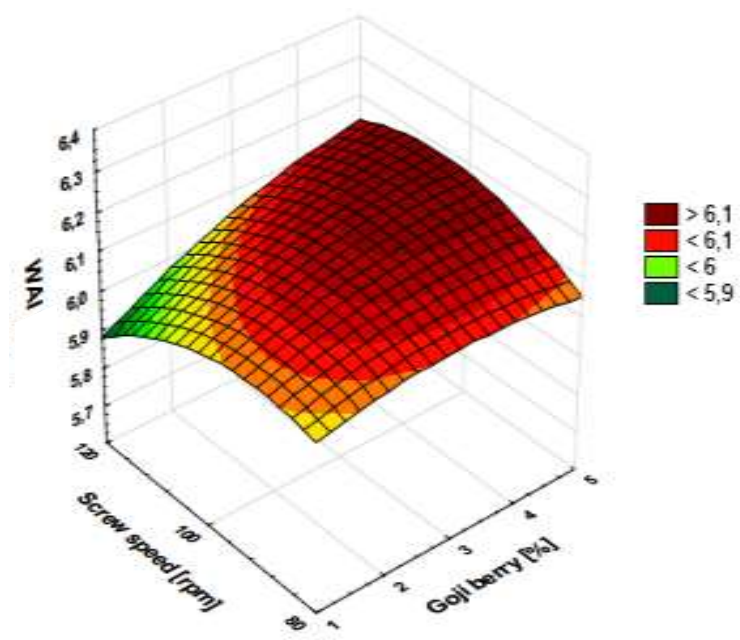

Fig. 1. Water absorption index (WAI) of instant gruels made from corn depending on goji berry content and screw speed applied during processing. 
In composition of the recipes of corn grits supplemented with $4-5 \%$ of goji berries it was observed the highest water absorption when the screw speed was $100 \mathrm{rpm}$ during the extrusion. The increasing amount of goji berries has shown insignificant effect on WAI values $\left(\mathrm{p}_{\text {-value }}=0.686\right)$ but the slight effect on water absorption growing was observed with increasing amount of dry fruits in the recipe, especially when 100 and $120 \mathrm{rpm}$ was applied during processing. Statistical analyses confirmed that variable screw speeds during the extrusion-cooking of instant gruels with goji berry addition have insignificant effect on water absorption of tested extrudates $\left(\mathrm{p}_{\text {-value }}=0.150\right)$.

WAI evaluated for instant gruels processed with the addition of cranberries attained values ranged from 5.86 to 6.25 and were comparable to corn-goji extrudates. The amount of cranberries $\left(\mathrm{p}_{\text {-value }}=0.474\right)$ and the screw speed applied $\left(\mathrm{p}_{\text {-value }}=0.167\right)$ have no significant effect on water absorption results. The highest results were observed if 2 and $3 \%$ of cranberries were added in the recipe (Fig. 2).

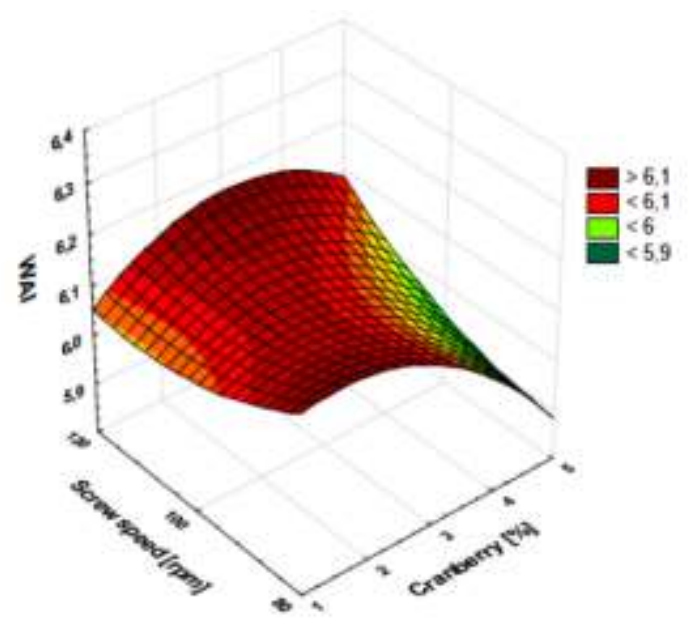

Fig. 2. Water absorption index (WAI) of instant gruels made from corn depending on cranberry content and screw speed applied during processing.

Cranberry addition affected on lower values of water absorption of instant gruels enriched with 4 and $5 \%$ of dry fruits content in the recipe when compared with the same amount of goji berry addition. It could be connected with the different composition of fruits used in the experiments.

\subsubsection{Water solubility index (WSI)}

The values of WSI of instant corn-goji berries gruels made with various screw speed during the extrusioncooking varied with the wide range from 14.76 to $21.14 \%$ (Fig. 3). Addition of dried goji fruits have no significant effect on components solubility in water ( $p$. value $=0.118$ ). Water solubility index was high in values when highest screw speed was applied during processing, but these dependencies were observed only if low amount of goji berries was used as an additive (up to $3 \%$ ). If higher amount of dried goji was added into the recipe the differences in water solubility were smaller. This could be connected with the application of high screw speed for processing of corn grits and thus intensive shearing treatment of starchy components resulting higher solubility. Increasing amount of pectinrich additive caused limitation of starch and increased amount of fibrous fractions from goji fruits able to keep the water (as showed by WAI results) and limiting the amount of soluble components passing into the water after centrifugation.

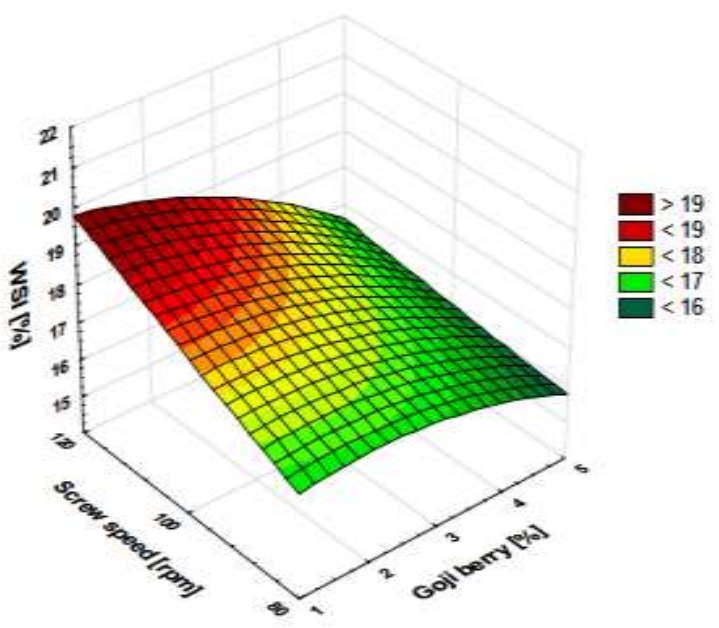

Fig. 3. Water solubility index (WSI) of instant gruels made from corn depending on goji berry content and screw speed applied during processing.

The WSI of instant gruels based on corn with the addition of cranberries was lower and reached values from 15.57 to $18.57 \%$. The cranberry fruits addition has similar effect as it was observed for goji berries. Increasing amount of dry cranberries in the recipe affected on lowering the water solubility of the extrudates.

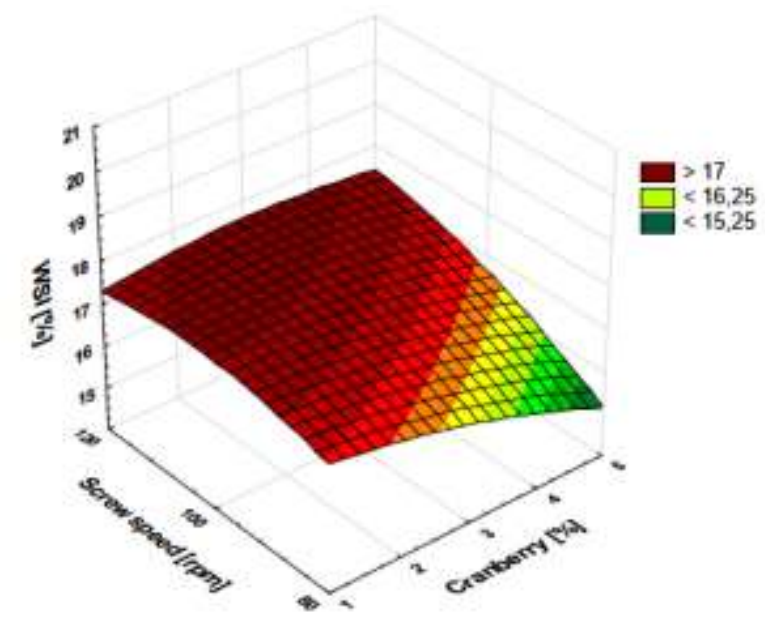

Fig. 4. Water solubility index (WSI) of instant gruels made from corn depending on cranberry content and screw speed applied during processing.

The highest solubility was evaluated for samples extruded at $120 \mathrm{rpm}$, especially when fruits addition not exceed $3 \%$, what could be the effect of the intensive thermomechanical treatment of ingredients during 
processing. At 80 and $100 \mathrm{rpm}$ decreasing trend was observed when 4 and $5 \%$ of additive was used, but generally cranberry addition has no significant effect on WSI values $\left(\mathrm{p}_{\text {-value }}=0.287\right)$. In general, also in this case the effect of screw speed on WSI was insignificant ( $\mathrm{p}_{\text {- }}$ value $=0.336$ ).

Water absorption index (WAI) and water solubility index (WSI) are two indices linked to the estimation of the behaviour of the processed material for application as a binder, a stabilizer, or emulsifier in various food systems $[3,5,8,10,36]$. WAI and WSI values extend in a wide range depending on the specific parameters applied during the extrusion-cooking process. Factors affecting the values of these indices can be classified into two main groups: the first group - linked to the raw materials, and the second one - includes parameters related to the extruder equipment and processing conditions. In particular, the first group refers to the initial composition of the raw material, the raw material formulation, the pre-processing treatments, the initial particle size of milled materials, and the milling procedure. The second group is related to the extruder conditions, such as extruder type, last section barrel temperature, or die temperature, feed moisture content, feed rate, screw speed, screw configuration, screw compression ratio, die dimension, and die configuration [36, 37]. Oikonomou and Krokida [11] proposed the mathematical model investigates the effect of die temperature, feed moisture content, and screw speed on the WAI (1.3 to 9.5$)$ and WSI (4.5 to $61.6 \%)$ properties for some extruded food products. They found that the model curves do not have a distinct slope as screw speed increases for all the extruded products. Similar trends have been observed in our study, especially when WAI results of enriched gruels were analysed.

\subsubsection{Color parameters}

The results of color characteristics evaluated for extrudates with fruits addition showed significant differences in CIE-Lab scale coordinates depend on the additive type and amount. The most significant $\left(\mathrm{p}_{\text {-value }}=\right.$ 0.000 ) was the effect of goji berry level on $L^{*}$ profile of instant gruels. Red in color goji berry fruits differ strongly from yellow corn grits so significant decrease in lightness was observed in supplemented gruels (Fig. 5). The $L^{*}$ values varied from 64.18 to 73.38 . There wasn't observed the effect of screw speed on gruels lightness ( $p$. value $=0.103)$. On the base of the results of $L^{*}$ measurement it can be concluded darker color of final products supplemented with the addition of goji berry than cranberries. Dried fruits were much darker than corn so also the extrudates characterized the lower lightness. $L^{*}$ values noted for instant gruels enriched with cranberries varied from 68.23 to 72.01 (Fig. 6).

The amount of cranberries showed significant effect $\left(\mathrm{p}_{\text {-value }}=0.000\right)$ on extrudates lightness, decreased $L^{*}$ values were observed when the higher amount of dry cranberries was used in the formula but differences were much lower than observed for samples with goji berry addition. In this case the screw speed applied during processing showed the significant effect $\left(\mathrm{p}_{\text {-value }}=0.022\right)$ on samples lightness. It was observed the slight decrease of lightness in extrudates processed with a higher screw speeds, especially when 1 and $2 \%$ of cranberries were added to the recipe.

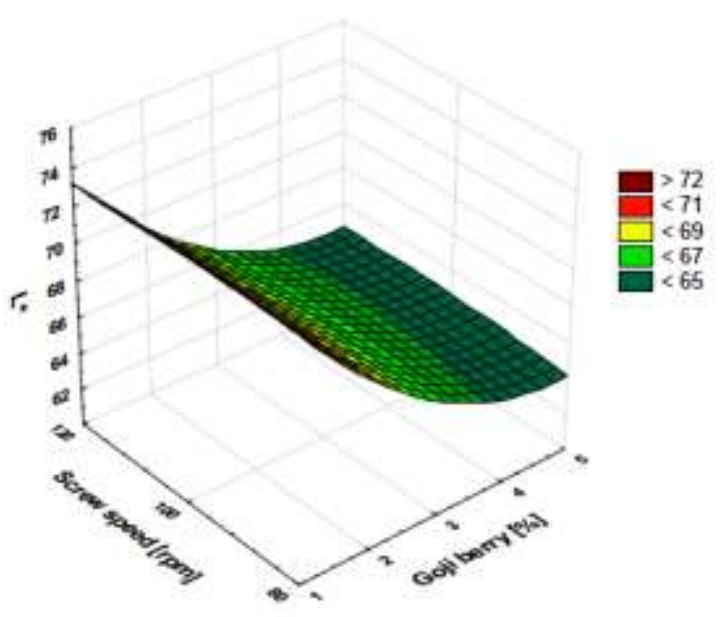

Fig. 5. $L^{*}$ profile of instant gruels made from corn depending on goji berry content and screw speed applied during processing.

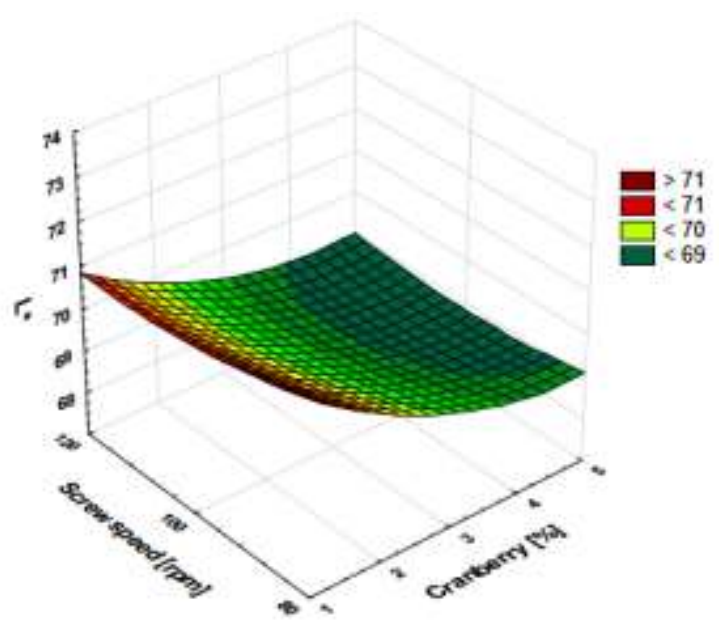

Fig. 6. $L^{*}$ profile of instant gruels made from corn depending on cranberry content and screw speed applied during processing.

On the figure 7 the red-green balance of instant gruels with goji berries is presented. The value of $a^{*}$ profile showed significant effect $\left(\mathrm{p}_{\text {-value }}=0.000\right)$ of fruits addition on increased red tint of extruded gruels. Addition up to $3 \%$ of goji berries in the recipe showed green tint presence, the most intensive for samples supplemented with $1 \%$ of goji dry fruits. Increasing the amount of additive resulted in formation of red tint with positive index values of a* profile ranged from -7.48 for samples with $1 \%$ of additive up to 3.74 for extrudates with $5 \%$ of goji berry in formula. This is the result of intensive red-orange color of dry goji berries and the additive increasing amount affected more red tint of instant gruels. The various screw speed applied during 
processing had no significant effect $\left(\mathrm{p}_{\text {-value }}=0.314\right)$ on that color coordinate.

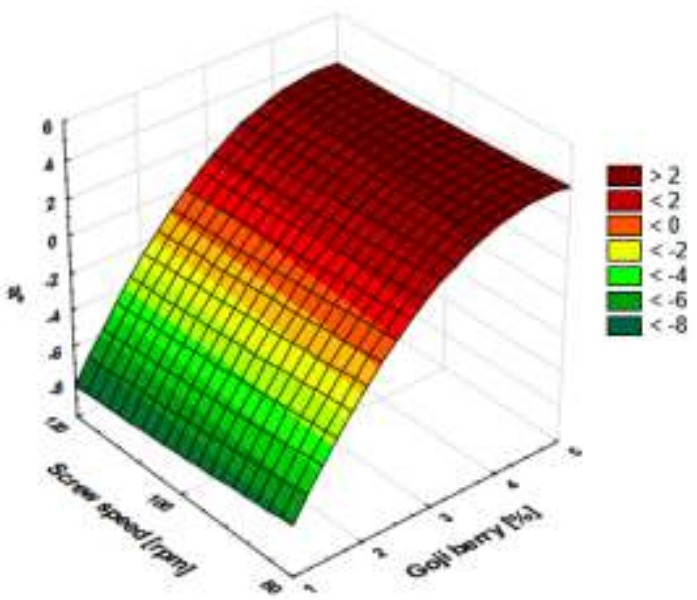

Fig. 7. Profile of $a^{*}$ balance of instant gruels made from corn depending on goji berry content and screw speed applied during processing.

The values of $a^{*}$ profile of instant gruels supplemented with the addition of cranberries gave negative values of this coordinate showing the green tint of extrudates with dry cranberries in the recipe (Fig. 8). The results varied from -6.62 to -3.19 and the differences were significant $\left(\mathrm{p}_{\text {-value }}=0.000\right)$. The most intensive greenes was observed for samples with $1 \%$ of cranberries and with incresing amount of fruits for 4 and $5 \%$ in the recipe lowered greeness intensity. Cranberries characterized red-brown color and extrudates showed lower reddness than observed for gruels supplemented with goji berries. Also for these samples no significant effect of screw speed was observed $\left(\mathrm{p}_{\text {-value }}=0.428\right)$.

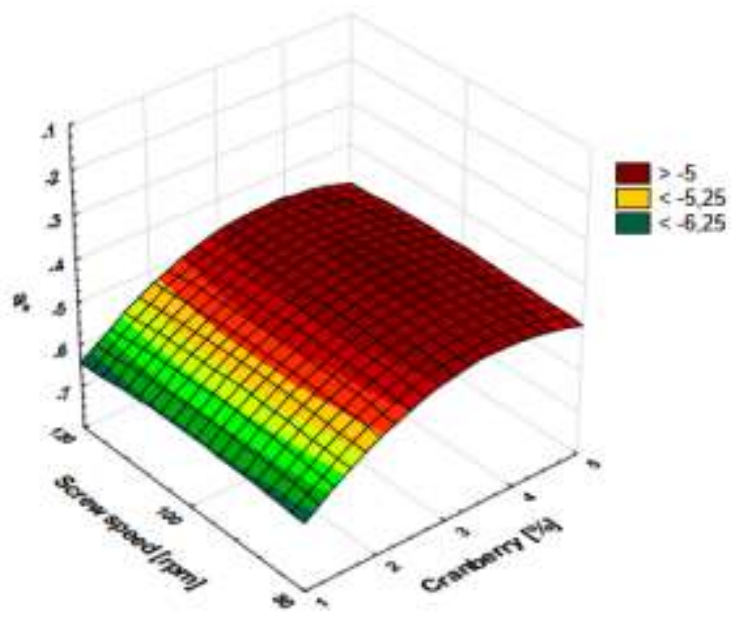

Fig. 8. Profile of $a^{*}$ balance of instant gruels made from corn depending on cranberry content and screw speed applied during processing.

Figure 9 illustrates the changes in blue-yellow balance of extruded gruels with the addition of goji berries. Values of $b^{*}$ coordinate reach the level of 63.98-68.37 and suggest intensive yellow tint of the extrudates. This color profile is connected with the intensive yellow color of corn grits used in the experiment and red-orange color of dry goji decreased yellowness of tested products. The effect of decreasing the yellowness was significant $\left(\mathrm{p}_{\text {-value }}=0.000\right)$ with an increase amount of goji fruits in the recipe. Test results showed slight but statistically significant effect ( $\mathrm{p}_{\text {-value }}=$ 0.013 ) of screw speed on $b^{*}$ values and decrease of yellowness was observed when higher screw speed was applied in the experiment.

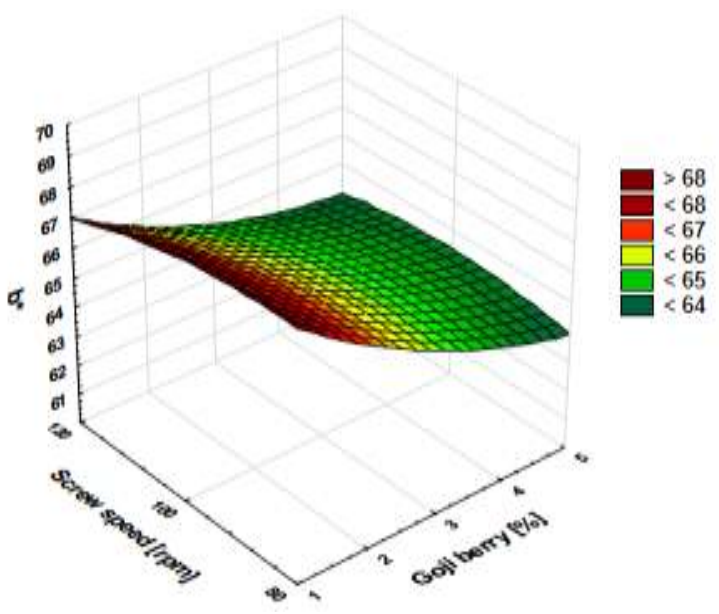

Fig. 9. Profile of $b^{*}$ balance of instant gruels made from corn depending on goji berry content and screw speed applied during processing.

Similar and more intensive tendency was observed for samples supplemented with cranberries (Fig. 10) but yellowness of tested samples was lower. The addition of dried cranberries showed significant effect $\left(\mathrm{p}_{\text {-value }}=\right.$ $0.000)$ on $^{*}$ values of extruded gruels. The range of yellowness varied from 64.38 if $1 \%$ of cranberries was used in the recipe up to 54.10 for gruels with the highest $5 \%$ of additive.

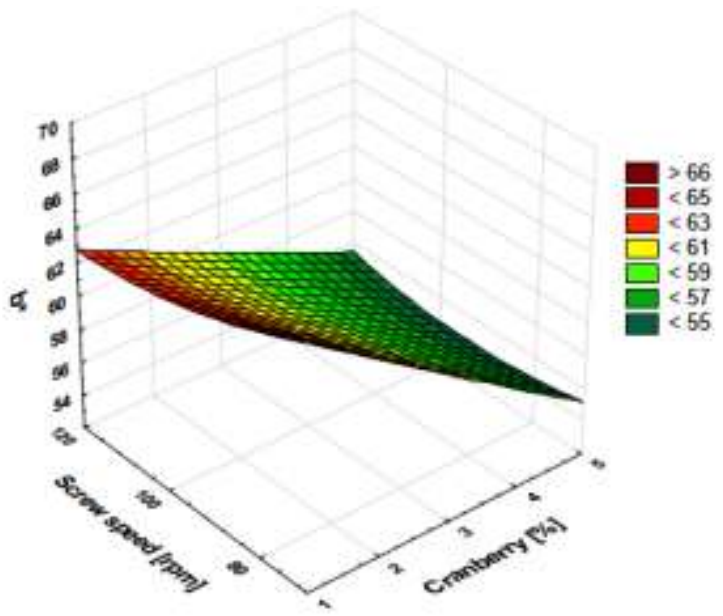

Fig. 10. Profile of $b^{*}$ balance of instant gruels made from corn depending on cranberry content and screw speed applied during processing.

Yellowness intensity in these samples was lower because of darker color of dry cranberries. The screw speed elevation during processing of samples with $1-4 \%$ of dry fruits addition decreased significantly $\left(\mathrm{p}_{\text {-value }}=\right.$ 0.022 ) the yellowness of tested samples and slight 
increase was observed for products extruded with 5\% of cranberries in formula with increasing the screw speed during processing.

\section{Conclusions}

The addition of dried fruits and screw speed applied during processing of instant corn-based gruels affected on the most of tested characteristics. Increasing amount of fruits and screw speed during the extrusion have no significant effect on WAI and WSI values. Significant effect of higher amount of goji berries and cranberries was observed for color coordinates evaluated with CIELab scale. Increasing amount of dry berries lowered lightness $L^{*}$ and yellowness of instant gruels, especially when goji berries were used in the recipe. Increasing the screw speed during processing decreased intensity of $b^{*}$ values.

\section{References}

1. P. Mayachiew, C. Charunuch, S. Devahastin, J. Food Sci. 80(12), E2782 (2015)

2. A. Altan, K.L. McCarthy, M. Maskan, J. Sci. Food Agric. 88,1648 (2008)

3. G. Bisharat, I. Katsavou, N. Panagiotou, M. Krokida, Z. Maroulis, Food Sci. Technol. Int. 21(8), 613 (2015)

4. E. Fleischman, R. Kowalski, C. Morris, T. Niguyen, C. Li, G. Ganjyal, C. Ross, J. Food Sci. 81(11), E2726 (2016)

5. D. Gbenyi, I. Nkama, M.H. Badau, J. Food Res. 4(2), 81 (2016)

6. A. Oniszczuk, A. Wójtowicz, T. Oniszczuk, M. Olech, R. Nowak, K. Wojtunik, M. Klimek, W. Krawczyk, M. Hajnos, Open Chem. 13, 1209 (2015)

7. M. Kręcisz, A. Wójtowicz, A. Oniszczuk Agriculture and Agricultural Science Procedia 7, 139 (2015)

8. M. Mitrus, L. Mościcki, Chem. Eng. Res. Des. 92, 778 (2014)

9. A. Wójtowicz, A. Kolasa, L. Mościcki, Pol. J. Food Nutr. Sci. 63(4) 239 (2013)

10. M.S. Alam, J. Kaur, H. Khaira, K. Gulpa, Crit. Rev. Food Sci. Nutr. 56(3), 445 (2016)

11. N.A. Oikonomou, Krokida, M.K., Int. J. Food Prop. 14, 199 (2011)

12. A. Oniszczuk, M. Olech, T. Oniszczuk, K. Wojtunik-Kulesza, A. Wójtowicz, Arabian J. Chem. (2016), doi:10.1016/j.arabjc.2016.09.003

13. R.P. Feliciano, C.G. Krueger, J.D. Reed, Mol. Nutr. Food Res. 59, 1292 (2015)

14. A. Stobnicka, M. Gniewosz, A. Miętuszewska, Bromat. Chem. Toksykol., XLIV(3), 650 (2011)

15. M.H. Grace, A. R. Massey, F. Mbeunkui, GG. Yousef, M.A. Lila, J. Food Sci. 77(8), H176 (2012)
16. D.L. McKay, J.B. Blumberg, Nutr. Rev. 65(11), 490 (2007)

17. C.C. Neto, Mol. Nutr. Food Res. 51, 652 (2007)

18. A. Basu, M. Rhone, T.J. Lyons, Nutr. Rev. 68(3), $168(2010)$

19. L. Gan, H. Zhang, X., Liang Yang, H. Bi Xu, Int. Immunopharmacol. 4, 563 (2004)

20. C. Bodet, F. Chandad, D. Grenier. J. Dent. Res. 85(3), 235 (2006)

21. S. Niro, A. Fratianni, G. Panfili, L. Falasca, L. Cinquanta, M.D. Rizvi Alam, Ital. J. Food Sci. 29 398 (2017)

22. P. Skenderidis, K. Petrotos, I. Giavasis, C. Hadjichristodoulou, A. Tsakalof, J. Food Process Eng. (2016) doi:10.1111/jfpe.12522

23. C.C. Wang, S.C. Chang, B.S. Inbaraj, B.H. Chen Food Chem., 120(1) 184 (2010)

24. S.C. Lam, Z. Luo, D.T. Wu, K.L. Cheong, D.J. Hu, Z.M. Xia, S.P. Li., J Food Sci 81(6), C1378 (2016)

25. J. Morais Ferreira, B. Azevedo, V. Luccas, H. Andre Bolini, J. Food Sci. 82(3), 818 (2017)

26. G-I. Hidalgo, M. Pilar Almajano, Antioxidants, 6, 7 (2017)

27. W.M. Tang, E. Chan, C.Y. Kwok, Y.K. Lee, J.H. Wu, C.W. Wan, R.Y. Chan, P.H. Yu, S.W. Chan, Int Inflammopharmacol. 20(6) 307 (2012)

28. B. Kulczyński, A. Gramza-Michałowska, Pol. J. Food Nutr. Sci. 66(2), 67 (2016)

29. M.K. Song, N.K. Salman, B.D. Roufogalis, T.H. W. Huang, Biochem. Pharmacol. 82(9) 1209 (2011)

30. Z. Shen, J. Wang, G. Li, Chinese J. Ophthalmol. 48(9) 824 (2012)

31. D. Cheng, H. Kong, Molecules, 16(3) 2542 (2011)

32. A. Wójtowicz, Acta Agroph., 11(2) 545 (2008)

33. ASAE Standard. ASAE S269.3. Wafers, pellet, and crumbles - definitions and methods for determining density, durability and moisture content (1989).

34. A. Wójtowicz, M. Mościcki, LWT-Food Sci. Technol., 59, 1175 (2014)

35. N.A. Oikonomou, Krokida M.K., Int. J. Food Prop. 15, 157 (2012)

36. D.R. Marques, Berwig K.P., Monteiro C.C. F., Oliveira D.M., Monteiro A.R.G., Chem. Eng. Trans. 57, 1921 (2017)

37. E. Lusac, Rooney L. Snack Foods Processing (CRC Press LLC, USA, 2002) 